\title{
Indigenous Healthcare: Determinants and Practices
}

\author{
Dr. Ramaiah Bheenaveni \\ Assistant Professor, Department of Sociology, Osmania University, Hyderabad-07. India
}

\begin{abstract}
India is the second most populous country in the world and having rich indigenous knowledge and healthcare systems to cure the sickness and diseases. Despite having modern medical facilities, most of the rural and tribal dwellers used to avail traditional or indigenous healthcare as the modern medicine is locally available, homemade and cost effective too. The fact is that privatization of modern medical-care has become costlier as the hospitals act like money spinning machines for the corporate sectors, thereby, the poor is unable to access such treatment. As a result, the folk isforced to visit the local faith healers or medicinal man to get rid of their diseases or health problems. Religious cohesiveness and solidarity conventionally admonish to promote traditonal kind of healthcare and herbal medicine. The poor implementation of healthcare programmes, low income, illiteracy, peer pressure, locality and cost effectiveness are the leadingenticements for the acceptance and adaptation of indigenous medicine. In fact, the reverse-modernity and thenostalgic notion of the masses are also considerable factors to tune with traditional medicine. With this conceptual framework, the present study has been conducted in Karimnagar District of Telangnga State with a total of 2,396 sample respondents who were selected on the basis of thecomputerized random method of sampling. Different qualitative and quantitative methods and tools were used for the collection of primary data and the analysis was done by the descriptive statistics.
\end{abstract}

Keywords:-Indigenous Healthcare, Folk Medicine, Rural Health, Traditional Healthcare, Health Determinates, Health Practices.

\section{INTRODUCTION}

The healthcare practices of the people not only preserve their health, but also affect all their day to day life. A man living in an interactive society is affected by what happens in his environment and how he reacts to it. That is, the deterrents and determinants of health and disease in a community cannot be completed unless the culture and healthcare practices of the people are considered (Willows, N. D. 2005). Many health practices usually help to perpetuate the prevalence of certain diseases and health problems in many parts of rural India (Rao, M. et al - 2011). Many have withstood and endured centuries of practice, and actually promote health. Some of these indigenous health practices have worked for the people who practice those(Mavalankar \& Prakasamma-2008). Unfortunately, they are not uncommon to people's mind to think of something crude, rudimentary and bad whenever they talk about healthcare practices as it concerns health.

It is relevant to state that not all indigenous/traditional health practices are bad to the people. Some have stood the test of time and have positive values, while others may be harmless, uncertain or negatively harmful (Balarajan, et al - 2011). They also believe it could minimize hospital expenses and could save money so that some believe there is a natural way of healing(Kishore et al - 2011). Nevertheless, the health beliefs and practices of community members have a profound effect on the health of the community. All people, irrespective of their caste and social status, have their own indigenous way of beliefs and practices concerning health and disease. Each society or community has its peculiar way of doing their things and it is known that these practices and beliefs go a long way in influencing the people's perception, attitude and management of diseases and other health related problems that befall them (Jorm, A. F. - 2000). Alternative medicine is also not generally regulated like indigenous and traditional medicine. Some forms of indigenous medicine are slightly regulated because they involve practices that fall under the jurisdiction of certain legal and governmental agencies, like Ayurveda (Bhalerao et al - 2013). However, the use of herbal medications is not regulated. It also observed that there is a growing interest in indigenous medicine (herbal medicine). People are noticing what a huge business healthcare is becoming. They are seeing that the driving force behind traditional medicine is no longer keeping people healthy, but also saving money and time. This has led many people to avoid buying into the traditional methods and instead they are opting for alternative methods (Robinson - 1978). Thus, it is essential to understand the awareness and practice of different indigenous and traditional healthcare practices of rural masses.And, it is alsoneeded that one should understand how and why each custom is practiced by any community if any meaningful impact is to be made towards maintaining sound health. 


\section{METHODS AND MATERIALS:}

The presentstudy has been carried out in Karimnagar district of Telangana State. It has basically followed survey-descriptive research design and examines the structure of indigenous beliefs and existing practices in relation to health and disease. The main emphasis of this study is on the traditional health practices and healing methods. It also analyses the impact ofthe modern healthcare system and how it affected the beliefs and practices of therural population. Further, it tries to bring out the rural health practices which can commonly be observed by the researcher and also traditionally continued from generations to generation. It used various methods of research, mainly by administrating the semi-structured interview schedule and partly by nonparticipant observation, focus group interview, ethnomethodology and ethnography. And, the participatory methods were used for health PRA of Ramachandrapur village located in Karimnagar district andthe case study method was used for both healers and patients.

The study concentrates on Karimnagar district which consists of 57 mandals and 1103 villages. The total rural population of the district i.e. $28,13,010$ of 1103 villages is considered asthe universe for this study as the scope of the study pertains to rural areas only. The sample 2,396 is taken for the study by following thecomputerized random method of sampling. Though, there are variations in health practices over all, the rural populationhas similar beliefs and practices in the district as same flora isfound in all parts of the district. The existence of traditional healers and social structure like caste, religion, economy, occupation, culture is similar. This makes the study a comprehensive understanding of whole with an appropriate sample. The homogeneity of the sample also assures that the results arenearer to facts. There is enough scope to do research in such areas as the dwindling traditional and indigenous healthcare knowledge demands an urgent attention. The individuals who depend on these practices also expressed their deep faith in indigenous healing systems.

\section{FINDINGS AND DISCUSSIONS}

The healthcare is no longer free, with rapidly rising costs, especially for serious illness. This will send many families into destitution while patients wait for death. When poor people talk about access to formal healthcare services, issues of cost and corruption repeatedly arise. For poor families who are already highly vulnerable, the cost of a sudden illness can be devastating, both because of lost income and because of the costs of treatment. While the actual treatment itself can be prohibitively expensive, in many cases there are other hidden costs that add to the overall financial burden of health care. These hidden costs include expenses incurred in traveling to a place where healthcare is available, and the psychological costs or stigma of having been treated for certain diseases. In addition to this, the access to healthcare is often heavily influenced by gender, with men and women using healthcare differently. The exercise of village health PRA denotes that the poor do not have any health consciousness and are not following strategies of health-maintenance. But they mostly visit traditional faith healers or formal hospitals only when they fall into sickness. This is not because they do not believe in or respect the curative powers of health providers but because their experience with health facilities has been so dismal over the past few years. Here, again theproblems stressed by poor include distance to healthcare facilities, lack of drugs, bribes and rude health personnel. Consequently, rural dwellers are forced to adopt the indigenous ways of healing systems. Besides, the indigenous and traditional health practices are still having prominence in rural areas as the modern medicine is expensive or inaccessible to them.

NGOs in Karimnagar district have limited presence and outreach. Where they are present NGOs are often praised as the only groups concerned about poor people. In the absence of government services, they have become important providers in bringing awareness on environmental sanitation and personal hygiene and the basic treatment patterns. In many places NGOs are clearly more trusted than the government. However there are also accounts of NGO ineffectiveness, irrelevance, and favoritism. Male roles in family healthcare are described as nominal among the rural dwellers. Women are typically the providers of healthcare rather than its recipients, and when resources are scarce they defer treatment of their own disorders in order to get care for their families. It is widely accepted that men are entitled to formal health care and the resources needed to secure it, long before women are entitled to such care. The responsibility for overall family healthcare particularly that of children, typically lies with women. Women in many households of backward communities will not take proper care of their own health and nutritional needs, which in turn affects their ability to feed the family and work. Poor women are often caught in a cycle of malnutrition and preventable diseases that stem directly from their place in the household. Children are among the most vulnerable segments of impoverished populations. Although there have been declines in infant mortality, poor children continue to suffer disproportionately from malnutrition, disease, and lack of adequate healthcare compared to their rich counterparts. Traditional healthcare practices are not only practiced inthe modern world but are also deeply respected for their easy reach and understanding of problems by faith healers and herbal practitioners. Expensive modern medicine with its prohibitive prices is also one more reason for non-interest in modern medicine in rural areas. Poverty is another major cause for choosing the indigenous health practices. Although 
the efficiency and affectivity of traditional medical practices are questioned by the modern allopathic practitioners there is no alternative for these poor. Also native wisdom lies in the emergency cases or trauma and its uses are limited. Sometimes diagnosis also may not be sophisticated as they mainly depend on experience. The true followers of the traditional medicine are rural population out of necessity and belief. The study outcomes are consistent with the earlier researches on similar topics. The main ethnobotonical studies have illustrated several herbs used in the Karimnagar and nearby districts. Other references are made from various sources by calculating their use in indigenous health care system. Of course certain herbs which are used in traditional health practices are upto the standards of modern herbal care. Others are kept as family secret or sometimethe only healer knows the ingredients used usually at the time of end older healer reveals. Sometimes that has been lost permanently without revealing tithe true nature of beliefs on health and decease one cannot access as they are dependent fundamentally on practitioners fame and the patient's faith over it. Although there are many cases of cure reported to what extent they are recordable is another question. They cannot be classified for the future generations and only through oral traditions they are preserved. Sometimes this knowledge is lost permanently withouta trace on kept throughthe tradition of generations to generation. The major problem with this system is many family members or one of them may not like to learn this system which will be a great loss for ever. As modern medicine penetrated into the younger generation there will be a survival crisis for these types of practices. The traditional herbal solutions are very effective in general if they are supported by years of experience and proper diagnosis with care. Sometimes what seems to be harmless like using bottle-gourd juice may be fatal if it has abitter taste when is consumed as it consists of poisonous chemicals. It is reported and written in the literature and news about such cases. One should not think all the herbals are safe and can be recommended for novices to do thesepractices. Some herbs may contain poison which should be administered with careful supervision of experienced herbalists and traditional healers who know correct dosage and way of intake. Though thisknowledgeis kept as top secret as the modern time requires it there is flexibility in revealing these secrets. It is the duty of responsible citizens to keep these treasures of traditional knowledge rather disposing of them by giving these practitioners status. Unlike in China where traditional herbal medicine has are aspectableplace and authorized and equal with modern medicine, in India there is a division and both systems exist side by side. Inthe case of India it is highly dividedpractices astraditional medicinal systems like Ayurveda, Sidha is not given importance on par with the Allopathy. Then we can imagine the fate of indigenous beliefs on health and disease. Either they will be treated suspiciously or as a complete trash. In this condition it is great opportunity to discuss the results of the present study and its prominence in changing conditions of twenty-first century medicinal practices.

\section{CONCLUSIONS}

\$ There is serious interest amongthe rural population in using traditional herbal medicinal practices. The lack of respect for them in modern society and lack of encouragement from the state leads the practioners not to pass this knowledge for the future generations.

* The traditional medicinal system like Ayurvedic, Sidda, and Unani are given less important and lack of funds are leading these practices into oblivion.

\$ There is no proper training for traditional healers in order to keep them inthe mainstream medicinal system.

\$ The people are consulting these indigenous practices when they cannot afford the modern medicine. This shows that rural poverty is the main cause of the dependency on the traditional healthcare system.

* The limbo of modern medicine is unreachable to the rural population and the compulsory dependence on thetraditional system is not a healthy sign for the both practioners.

\$ It has also been observed that traditional beliefs like Vastu, Magic, Mantra and Tantra also have a deep impact on the healthcare beliefs and conditions of the health of the rural people.

\$ The religious belief and social stratification also place a major role in keeping the rural health and also their preference for modern or indigenous healing system.

\$There is a positive relationship between native/local knowledge and indigenous practices of a given.

\$The impact of various social factors such as caste, religion, traditional customs and occupation has been observed with regard to indigenous health practices.

4 The faith in indigenous health practices is proportionate to the social factors among which, the important ones are caste, education, religion, culture and customs.

\$ The validity of the traditional knowledge depends on long term experiences of rural population vis-à-vis traditional medicinal practices.

\$It is not that all indigenous health practices are based on social facts but on non-social facts such as faith in herbs, traditional medicinal instruments which drive the followers towards them regardless of their wishes.

\$The rise of modern medicine led to theawakening of indigenous health practices which are partly lying in the economic and cultural settings of a given society. 


\section{SUGGESTIONS}

\$ The state should recognize the importance of traditional practices wherever required and utilize them effectively in order to maintain health and by following 'health for all'.

\$ The traditional knowledge should be recorded and written for the benefit of future generations.

\$There should be a serious attempt adopting indigenous health practices in the regular healthcare system of therural population.

\$ The state should certify and clarify traditional healers into some kind of medical system which can serve the rural population in order to meet their needs.

\$ One should separatethe chaff from the grain in traditional healthcare practices so that useful practices can be kept aside for the benefit ofthe community and harmful practices kept at bay.

\$One should discourage those practices which are superstitious and harmful to the patients. Conceiving how they damage individual health. Here, one should adopt the healthcare either traditional or modern based on observable facts.

\$ AYUSH department must be given responsibility to recognize these traditional healthcare practioners in order to regularize their service to the masses and also avoid quacks inthe indigenous healthcare system.

\$ There should bean honorarium for genuine traditional healers in order to keep them in the profession as well as by offering benefits to reveal their secret practices making them as common knowledge.

\$Each district should be allotted land for the department of medicinal plants in order to keep these indigenous herbal practices.

\$ Masses should be educated to use herbal medicines and its cautions as some of them may cause severe damage, if not used under professional and expert guidance.

\$There should be district level survey of all type of indigenous healthcare practices and record them for the benefit of thewhole population of state so that this knowledge can spread to other regions also.

* The Central government published botanical medicinal plant data, these indigenous herbals should be included for the benefit of the nation as well as the export of herbals.

\$It is better if the state or a well-equipped NGO publish the bulletin on indigenous herbs and their usages.

* All indigenous knowledge should be given geographical identity and patented in the name of respective community where they originated in order to keep this knowledge falling away from unscrupulous, multinational companies and drug industry as there are many cases reporting that they were patented in the name of individuals and companies. For example, Neem, Turmeric, Vempali, Basmati, etc.

\section{REFERENCES}

[1] Balarajan, Y., Selvaraj, S., \& Subramanian, S. V. (2011). Health care and equity in India. The Lancet, 377(9764), 505-515.

[2] Bhalerao, M. S., Bolshete, P. M., Swar, B. D., Bangera, T. A., Kolhe, V. R., Tambe, M. J., ... \& Thatte, U. M. (2013). Use of and satisfaction with complementary and alternative medicine in four chronic diseases: a cross-sectional study from India.

[3] Jorm, A. F. (2000). Mental health literacy: Public knowledge and beliefs about. British Journal of Psychiatry, 177, 396-401.

[4] Kishore, J., Gupta, A., Jiloha, R. C., \& Bantman, P. (2011). Myths, beliefs and perceptions about mental disorders and health-seeking behavior in Delhi, India. Indian journal of Psychiatry, 53(4), 324.

[5] Mavalankar, D., Vora, K., \& Prakasamma, M. (2008). Achieving millennium development goal 5: is India serious? Bulletin of the World Health Organization, 86(4), 243-243A.

[6] Rao, M., Rao, K. D., Kumar, A. S., Chatterjee, M., \& Sundararaman, T. (2011). Human Resources for Health in India. The Lancet, 377 (9765), 587-598.

[7] Robinson, David. (1978). Patients, Practitioners and Medical Care: Aspects of Medical Sociology, Cox \& Wyman Ltd, London.

[8] Willows, N. D. (2005). Determinants of healthy eating in Aboriginal peoples in Canada: the current state of knowledge and research gaps. Canadian Journal of Public Health/Revue Canadienne de Sante'e Publique, S32-S36. 